\title{
Wild Isolates of Neurospora crassa Reveal Three Conidiophore Architectural Phenotypes
}

\author{
Emily K. Krach ${ }^{1}$, Yue Wu ${ }^{2}$ (D) Michael Skaro ${ }^{2}$, Leidong Mao ${ }^{3}$ and Jonathan Arnold ${ }^{1,2, *(D)}$ \\ 1 Genetics Department, University of Georgia, Athens, GA 30602, USA; ekrach@uga.edu \\ 2 Institute of Bioinformatics, University of Georgia, Athens, GA 30602, USA; yue.wu@uga.edu (Y.W.); \\ Michael.skaro@uga.edu (M.S.) \\ 3 School of Electrical and Computer Engineering, College of Engineering, University of Georgia, \\ Athens, GA 30602, USA; mao@uga.edu \\ * Correspondence: Arnold@uga.edu
}

Received: 30 September 2020; Accepted: 6 November 2020; Published: 9 November 2020

\begin{abstract}
The vegetative life cycle in the model filamentous fungus, Neurospora crassa, relies on the development of conidiophores to produce new spores. Environmental, temporal, and genetic components of conidiophore development have been well characterized; however, little is known about their morphological variation. We explored conidiophore architectural variation in a natural population using a wild population collection of 21 strains from Louisiana, United States of America (USA). Our work reveals three novel architectural phenotypes, Wild Type, Bulky, and Wrap, and shows their maintenance throughout the duration of conidiophore development. Furthermore, we present a novel image-classifier using a convolutional neural network specifically developed to assign conidiophore architectural phenotypes in a high-throughput manner. To estimate an inheritance model for this discrete complex trait, crosses between strains of each phenotype were conducted, and conidiophores of subsequent progeny were characterized using the trained classifier. Our model suggests that conidiophore architecture is controlled by at least two genes and has a heritability of 0.23. Additionally, we quantified the number of conidia produced by each conidiophore type and their dispersion distance, suggesting that conidiophore architectural phenotype may impact $N$. crassa colonization capacity.
\end{abstract}

Keywords: Neurospora crassa; natural variation; complex trait; conidiophore development; phenomics; convolutional neural network; generalized linear model; spore shadow

\section{Introduction}

Neurospora crassa propagates asexually through the dissemination of haploid spores, conidia, which develop via specialized aerial structures called conidiophores [1]. Macroconidiophores give rise to macroconidia and are morphologically and developmentally distinct from their smaller counterparts, microconidiophores, which give rise to uninuclear microconidia [2]. Since macroconidiophores are the subject of this work, they are hereafter referred to simply as conidiophores. Development of conidiophores is under strict environmental and temporal control, requiring cues such as desiccation, nutrient deprivation, and light exposure for its induction [3]. After exposure to these environmental triggers, aerial hyphae grow perpendicular to the preceding mycelial mat to stimulate conidiophore development. The organism subsequently undergoes a series of constriction budding followed by crosswall formations until eventual sporulation, roughly 10 hours (h) following the beginning of this process [4]. It is through this dissemination of conidia that the vegetative life cycle can propagate, thus allowing new filaments to germinate after a period of dormancy. Conidiophore development is under strict circadian control, requiring activation of the white collar complex (WCC) by light for 
activation of $f l u f f y(f l)$, a necessary regulator of conidiophore development $[5,6]$. While environmental, temporal, and genetic components of conidiophore development in $N$. crassa have been well characterized [7], little is known about morphological variation of these structures, particularly in natural populations.

In other filamentous fungal species, conidiophore architecture has been shown to directly impact the ability of an organism to disseminate throughout an environment and infect host tissues. For example, conidiophore morphology is altered in Aspergillus niger following deletion of velvet $A$, directly impacting both spore dispersion and colonization capacity of the organism [8]. Additionally, mutating the acropetal locus of Magnaporthe grisea modifies arrangement of the developing spores in a conidiophore, resulting in spores that are nonpathogenic [9]. Given these known effects in other fungal species, we sought to characterize natural conidiophore architectural variation in N. crassa, estimate heritability of these phenotypes, and examine their potential impact on sporulation. To do this, we employed a wild population collection of $21 \mathrm{~N}$. crassa strains collected from Louisiana, United States of America (USA) [10]. These isolates have been used in previous population genetics studies to elucidate local adaptations to cold tolerance and circadian rhythm [11] and characterize novel functions of loci $[12,13]$. Our work reveals three novel and distinct conidiophore architectural phenotypes among these wild populations. These phenotypes persist throughout the duration of conidiophore development and are described here as Wild Type (WT), Bulky, and Wrap. By conducting crosses between each phenotype and characterizing subsequent progeny conidiophores, we were able to fit a model for heritability of the discrete complex trait, conidiophore type. Our model suggests that at least two genes control conidiophore architectural phenotype and estimates a heritability of 0.23 . We explored the potential impact of conidiophore phenotype on sporulation by quantifying the number of conidia produced and their dispersal distance. Furthermore, we present a novel approach with an accurate image-classifier using a convolutional neural network [14] specifically developed to assign conidiophore architectural phenotypes in a high-throughput manner.

\section{Materials and Methods}

\subsection{Strains and Media}

Wild Louisiana isolates were obtained from the Fungal Genetics Stock Center (FGSC, Manhattan, KS, USA) [15] and are listed in Table A1 (Appendix A). Strains were maintained on $1.8 \%$ glucose $/ 1.8 \%$ fructose $/ 1.5 \%$ agar slants with $1 \times$ Vogel's media and recommended biotin and trace element supplements. To isolate conidiophores, strains were inoculated on $1.8 \%$ glucose $/ 1.8 \%$ fructose $/ 1.5 \%$ agar plates $(100 \times 15$ millimeters $(\mathrm{mm}))$ with $1 \times$ Vogel's media, biotin, and trace element supplements and incubated at $30^{\circ} \mathrm{C}$ for $20 \mathrm{~h}$. Cultures were then harvested onto a $82 \mathrm{~mm}$ diameter nitrocellulose membrane with 0.45 micron $(\mu \mathrm{m})$ pore size (Whatman Protran BA-85, Maidstone, England), inverted onto a new agar plate as described above, and placed under light for aerial hyphae to penetrate the membrane. After $20 \mathrm{~h}$, membranes were removed from the agar and secured on a flat surface for imaging of conidiophores. Method was adapted from Bailey-Shrode and Ebbole, 2004 [6].

Cultures for time-course images were grown following the same protocol with nitrocellulose membranes removed at 20, 25, or $35 \mathrm{~h}$ for imaging of conidiophores at different stages of development.

\subsection{Crosses and Progeny Screening}

Crosses were performed in the dark on cornmeal crossing medium, after which ascospores were plated on sorbose + fructose + glucose (SFG) media. Colonies were subsequently picked to isolate random ascospore progeny. Strains selected for crossing were FGSC8872 (WT) $\times$ FGSC3943 (Bulky), FGSC8872 (WT) × FGSC8876 (Wrap), and FGSC2229 (Bulky) × FGSC8876 (Wrap). Crosses were conducted in duplicate with 25 progeny selected from each. To isolate and image conidiophores in a high-throughput manner while preventing fusion of different progeny sharing a plate, each strain was inoculated on a $1 \mathrm{~mL} 1.5 \%$ agar droplet containing $1.8 \%$ glucose $/ 1.8 \%$ fructose with $1 \times$ Vogel's media 
and recommended biotin and trace element supplements. Each $150 \times 15 \mathrm{~mm}$ petri dish contained eight agar droplets evenly spaced roughly $2.5 \mathrm{~cm}$ apart. Each droplet was inoculated with progeny conidia and incubated at $30^{\circ} \mathrm{C}$ for $20 \mathrm{~h}$ to allow sufficient mycelial growth without hyphal fusion between droplets. Each droplet was then harvested onto a separate nitrocellulose membrane with $0.45 \mu \mathrm{m}$ pore size. Each membrane was inverted onto a new agar droplet as described above and placed under light for aerial hyphae to penetrate the membrane. After $25 \mathrm{~h}$, membranes were removed from the agar and secured on a flat surface for imaging of conidiophores.

\subsection{Microscopy and Image Deconvolution}

Nitrocellulose membranes containing conidiophores were visualized on an inverted microscope (Axio Observer A1, Carl Zeiss Microscopy, LLC, Thornwood, NY, USA) at 20× magnification and brightfield images were taken with a charge-coupled device (CCD) camera (AxioCam HRm, Carl Zeiss Microscopy, LLC, Thornwood, NY, USA). Multiple z-slices were captured and overlaid in ImageJ [16] to convey a complete representation of each three-dimensional structure. Augmentation including contrast enhancement and noise and background subtraction was conducted on image stacks to isolate conidiophores from underlying mycelia and/or aerial hyphae [17].

\subsection{Automated Phenotype Classification}

Residual nets (ResNet) was used to classify brightfield images into the three phenotypic classes by transfer learning [14]. After some hyperparameter searching, ResNet-50 pretrained on ImageNet was chosen as the starting neural network for image classification [18]. Training parameters were as follows: batch size, 15; initial learning rate, 0.001; stochastic gradient decent as the optimizer. The training process did not update parameters before module 3 in ResNet, and the parameters were only updated for the last 27 convolutional layers and the fully connected layer. Learning rates decayed when plateau was used for modifying learning. Augmentation including random rotation, random horizontal flip, and random gray was introduced in the training to simulate realistic diversity and prevent overfitting. Before augmentation, all images went through padding and resize to fit the ResNet input size. All images were converted to tensor and normalized.

We balanced the three classes of conidiophore phenotypes and separated the dataset into training (543), validation (66), and test sets (66). An external test set generated from progeny conidiophore images was collected in a separate batch and used for further validation (WT: 40; Bulky: 60; Wrap: 49). The training set was used to train the neural network and update its parameters. The validation set was used for updating learning rates and finding the final model to alleviate overfitting (Figure A1 in the Appendix A). The test set was not used until the final performance evaluation. We calculated accuracy and macro definition of precision and recall for multiclass classification [19]. The model training and inference used PyTorch [20], torchvision, and CUDA. P100 at Sapelo2 of GACRC was used for model training.

Feature importance was also evaluated on the test set. Features were evaluated on an integrated gradient [21], and the noise tunnel method [22] smoothed the result. We chose the maximal value as baseline instead of regular choices (constant black) because, in our brightfield images, a lighter background indicated the absence of features [23]. In noise tunnel, the noise was added 10 times, and the means of squared attributions were used.

The codes for training and visualization are shared on GitHub (https://github.com/michaelSkaro/ image_classification/tree/master/src).

\subsection{Sporulation Quantification}

Representative strains for each phenotypic class (FGSC8872, FGSC8876, and FGSC3943) were inoculated on $1.8 \%$ glucose $/ 1.8 \%$ fructose $/ 1.5 \%$ agar plates with $1 \times$ Vogel's media, biotin, and trace element supplements and incubated at $30^{\circ} \mathrm{C}$ for $30 \mathrm{~h}$. Three biological replicates of each strain were performed. Cultures were then harvested onto an $82 \mathrm{~mm}$ diameter nitrocellulose membrane and 
inverted onto a new agar plate as previously described above. Plates were placed under light for $32 \mathrm{~h}$ to allow penetration of aerial hyphae, development of conidiophores, and subsequent sporulation. Nitrocellulose membranes were then removed, and conidia were suspended into $50 \mathrm{~mL}$ of water and counted and sized with the Cellometer Auto 2000 (Nexcelom, Inc., Lawrence, MA, USA) [24,25].

To compare dispersal distance traveled by spores of each phenotype, the same representative strains listed above were inoculated onto the same growth medium. After $20 \mathrm{~h}$ at $30^{\circ} \mathrm{C}$, cultures were harvested onto a $60 \mathrm{~mm}$ diameter nitrocellulose membrane, inverted onto a new plate, and set under light for $30 \mathrm{~h}$. Membranes were then removed and placed at the center of a $245 \mathrm{~mm}$ square bioassay dish, where SFG medium surrounded a $60 \mathrm{~mm}$ diameter blank space now occupied by the membrane. The dish was placed under light for $48 \mathrm{~h}$ to allow for sporulation and subsequent colonial growth. At this time, pictures of each plate were taken with an iPhone XS, and contrast enhancement was performed in ImageJ. ImageJ was used to measure distance from the center of each nitrocellulose membrane to the center of each colony. Two biological replicates were conducted for each strain.

\section{Results}

\subsection{Wild N. crassa Isolates Exhibit Three Conidiophore Architectural Phenotypes}

Conidiophores from 21 wild Louisiana populations of N. crassa (Table A1) were isolated and imaged, and we identified striking architectural variation both within and between populations. Conidiophores were classified by their morphology into three groups that are hereafter referred to as Wild Type (WT), Bulky, and Wrap. The WT phenotypic class is characterized by linear chains of developing conidia that extend and branch outward from the aerial hypha. Developing spores in Bulky conidiophores, however, form crowded clusters that inhibit the ability to distinguish linear chains. These spores also display more variation in their size and shape. In the Wrap phenotype, conidia cling to and/or wrap around a hyphal filament rather than extending and branching outward as in the other phenotypic groups (Figure 1).
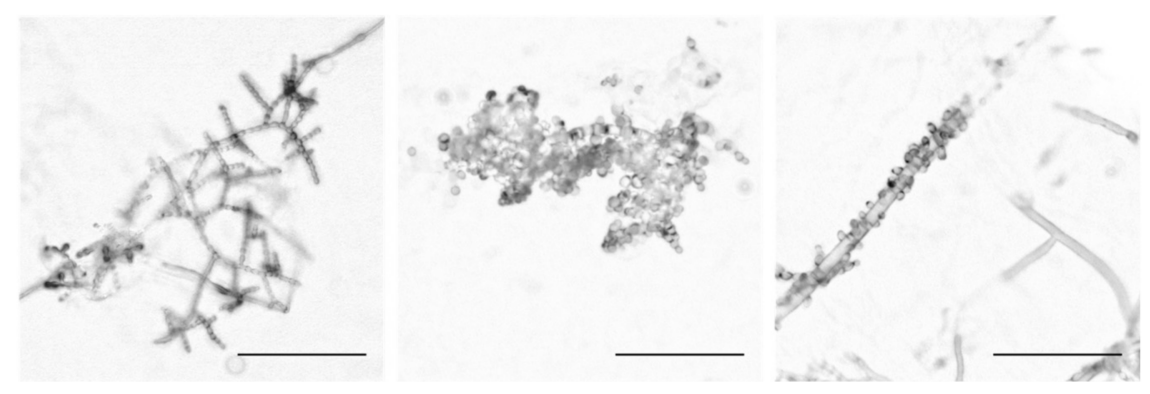

Figure 1. The three conidiophore architectural phenotypes. The Wild-Type (WT) phenotype is depicted in the first panel with FGSC2489, followed by the Bulky phenotype with FGSC8878, and Wrap with FGSC8876. Scale bar, 100 microns $(\mu \mathrm{m})$.

Each brightfield image was manually classified into one (or two) of the phenotypic classes using the guidelines described above (Table 1). Many strains had one phenotype demonstrating a clear majority, although every population had representation from at least two of the three phenotypes. All strains developed conidiophores with WT and Wrap phenotypes. Most strains also had Bulky conidiophores, with the exception of five populations (FGSC0847, FGSC2222, FGSC2228, FGSC3199, FGSC8872) lacking such individuals. Among the population set of 21 wild strains, 13 showed a majority of WT conidiophores, while five had a majority of Bulky, and three had a majority of Wrap. 
Table 1. Classifications of conidiophores belonging to each wild isolate (chi-squared test for independence: $\left.X^{2}=380.92, \mathrm{df}=40, p<2.2 \times 10^{-16}\right)$. Individual conidiophore counts vary with each strain. Phenotypes are balanced for classifier training described below.

\begin{tabular}{ccccc}
\hline Strain & WT & Bulky & Wrap & Total \\
\hline FGSC0847 & 22 & 0 & 4 & 26 \\
FGSC2221 & 26 & 1 & 3 & 30 \\
FGSC2222 & 13 & 0 & 8 & 21 \\
FGSC2223 & 10 & 2 & 3 & 15 \\
FGSC2224 & 16 & 23 & 6 & 45 \\
FGSC2228 & 39 & 0 & 5 & 44 \\
FGSC2229 & 14 & 54 & 48 & 116 \\
FGSC2489 & 32 & 7 & 1 & 40 \\
FGSC3199 & 17 & 0 & 2 & 19 \\
FGSC3200 & 30 & 5 & 23 & 58 \\
FGSC3211 & 20 & 16 & 7 & 43 \\
FGSC3212 & 54 & 2 & 6 & 62 \\
FGSC3943 & 32 & 80 & 52 & 164 \\
FGSC8870 & 19 & 2 & 9 & 30 \\
FGSC8871 & 16 & 6 & 17 & 39 \\
FGSC8872 & 14 & 0 & 4 & 18 \\
FGSC8873 & 8 & 11 & 13 & 32 \\
FGSC8874 & 20 & 3 & 14 & 37 \\
FGSC8876 & 15 & 21 & 42 & 78 \\
FGSC8878 & 20 & 26 & 12 & 58 \\
FGSC8879 & 6 & 12 & 11 & 29 \\
Total & 443 & 271 & 290 & \\
\hline
\end{tabular}

\subsection{Architectural Phenotypes Are Consistent throughout Conidiophore Development}

To ensure the three phenotypes were not just representations of different time points along the same developmental trajectory, we captured images of strains strongly representing each phenotype at 20, 25, 30 , and $35 \mathrm{~h}$ after transferring cultures to the nitrocellulose membrane, with $30 \mathrm{~h}$ as the initial reference point for mature conidiophore images. It is important to note that each time point was collected from a separate set of cultures, and these are not time-series images. WT, Bulky, and Wrap phenotypes were presented as early as $20 \mathrm{~h}$ and maintained until sporulation captured at $35 \mathrm{~h}$, suggesting that the three architectural phenotypes are not a result of temporal disparity along the same developmental trajectory (Figure 2).
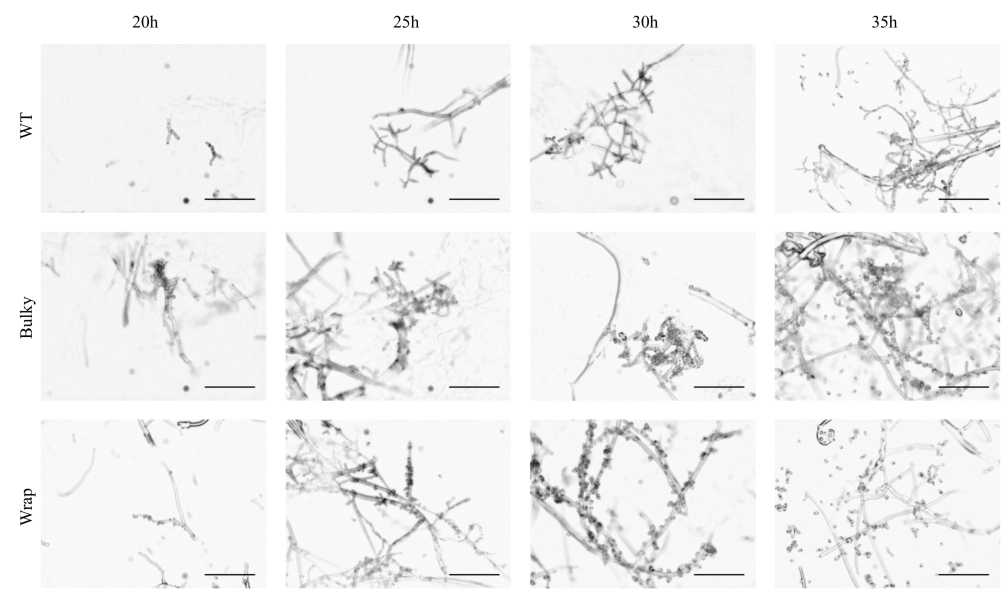

Figure 2. Development of conidiophore architectural phenotypes over time. Individual cultures were imaged at 20, 25, 30 and $35 \mathrm{~h}$ after being transferred to a nitrocellulose membrane and placed under light. Development of the conidiophore begins at roughly $20 \mathrm{~h}$ and concludes with sporulation by 35 h. Representative strains used were as follows: WT, FGSC2489; Bulky, FGSC8879; Wrap, FGSC8876. Scale bar, $100 \mu \mathrm{m}$. 


\subsection{There Is No Dependence of Phenotype on Strain Collection Environment}

Next, we investigated whether or not there was a correlation between the environment from which a strain was collected and its most prominent conidiophore phenotype. We found no statistically significant dependence of phenotype on collection substrate as reported by the Fungal Genetics Stock Center (FGSC) [15] (chi-squared test for independence; $X^{2}=4.9196, \mathrm{df}=10, p=0.8965$ ) (Figure 3). Interestingly, all strains with a majority of Wrap conidiophores $(n=3)$ were collected from sugarcane, although WT and Bulky strains were also found on this substrate. WT strains were found on all six substrates (sugarcane, grass burn, burned stump, pine burn, bonfire, and unknown), and Bulky strains were found on sugarcane, grass burn, and pine burn. We also did not find any clear relationship between most prominent phenotype and the town from which each strain was collected, as reported by FGSC (Figure 4).

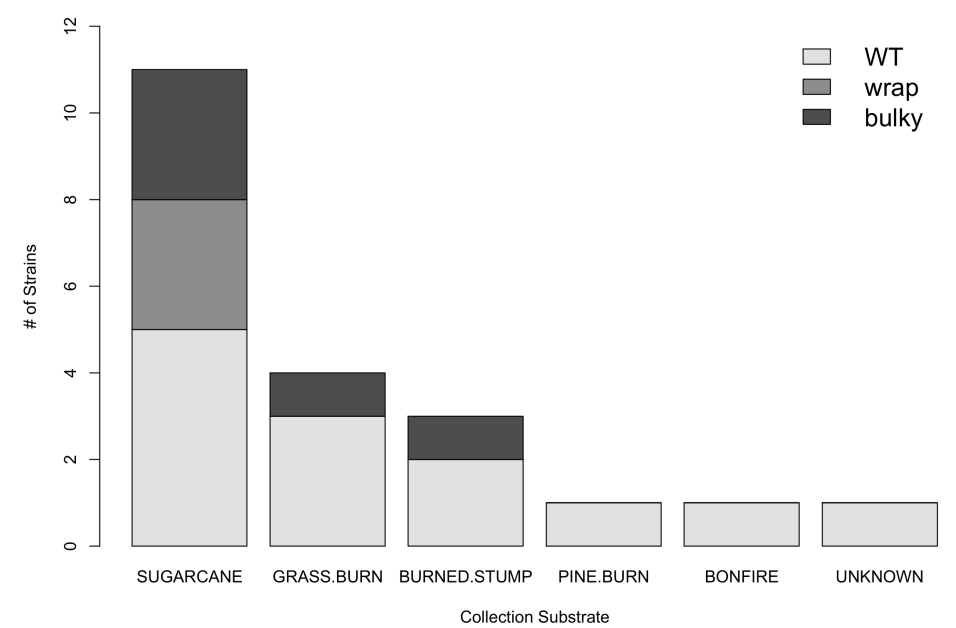

Figure 3. There is no significant correlation between the substrate from which a strain was collected and its most prominent phenotype $\left(X^{2}=4.9196, \mathrm{df}=10, p=0.8965\right)$ from Table 1 . Collection substrate as reported by the Fungal Genetics Stock Center (FGSC).

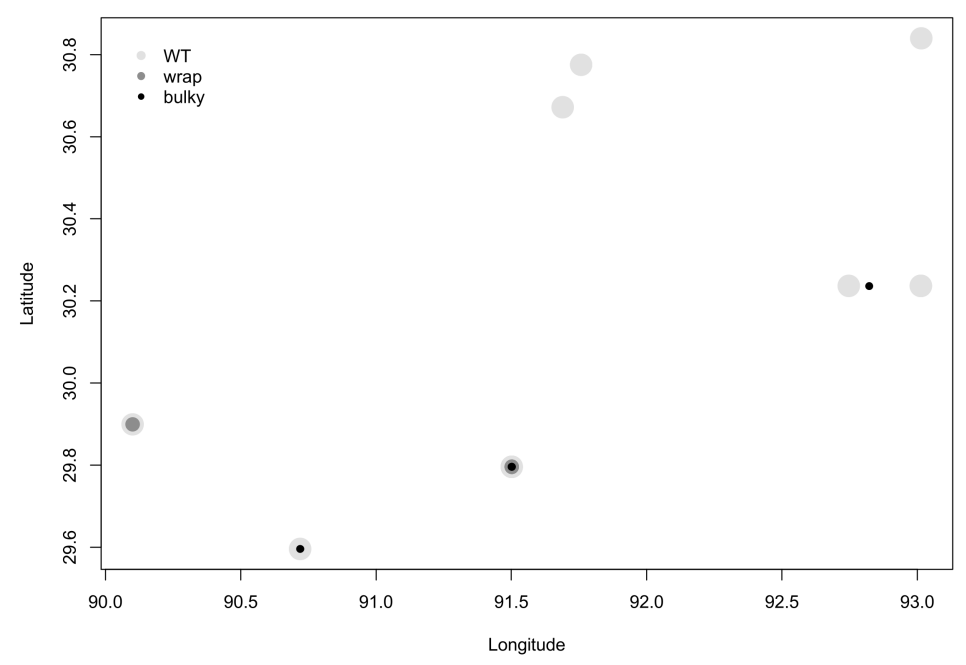

Figure 4. There is no clear relationship between most prominent phenotype and collection site. Louisiana town names were reported by the FGSC and are depicted here as geographical coordinates. Overlapping datapoints depict multiple strains collected from the same town. 


\subsection{Architectural Phenotypes Can Be Automatically Classified and Corresponding Features Can Be Extracted}

Manually categorizing conidiophores into phenotypic classes is time-consuming and introduces potential bias. To streamline the future classification of additional conidiophore images, an automated classification process was developed. We demonstrated that an accurate image classifier could be constructed based on the limited conidiophore image dataset of 543 training samples and presents reasonable performances (Tables 2 and 3).

Table 2. Performance of ResNet-50 classification on training, validation, and test sets. Accuracy, precision, and recall are used for evolution.

\begin{tabular}{cccc}
\hline & Accuracy & Precision & Recall \\
\hline Training & 0.9632 & 0.9644 & 0.9632 \\
Validation & 0.7879 & 0.7890 & 0.7879 \\
Test & 0.7576 & 0.7540 & 0.7576 \\
External test set & 0.6779 & 0.6802 & 0.6639 \\
\hline
\end{tabular}

Table 3. Confusion table for the test set. Each row indicates different true labels, and each column indicates different predictions. In the test set, the accuracies are 0.9545 (Bulky), 0.5909 (Wrap), and 0.7273 (WT).

\begin{tabular}{cccc}
\hline & Bulky & Wrap & WT \\
\hline Bulky & 21 & 1 & 0 \\
Wrap & 4 & 13 & 5 \\
WT & 2 & 4 & 16 \\
\hline
\end{tabular}

Images from both the test and the validation set can be accurately classified at rates of $76 \%$ and $79 \%$, respectively (Figure 5). Testing on an external dataset from a separate batch showed slightly worse performance at $68 \%$ accuracy. Feature importance is also evaluated (Figure 5), confirming that the program is properly identifying the conidiophore within an image for classification. This method was used to classify images of progeny conidiophores in a high-throughput manner to estimate potential heritability of these phenotypes, as described below.

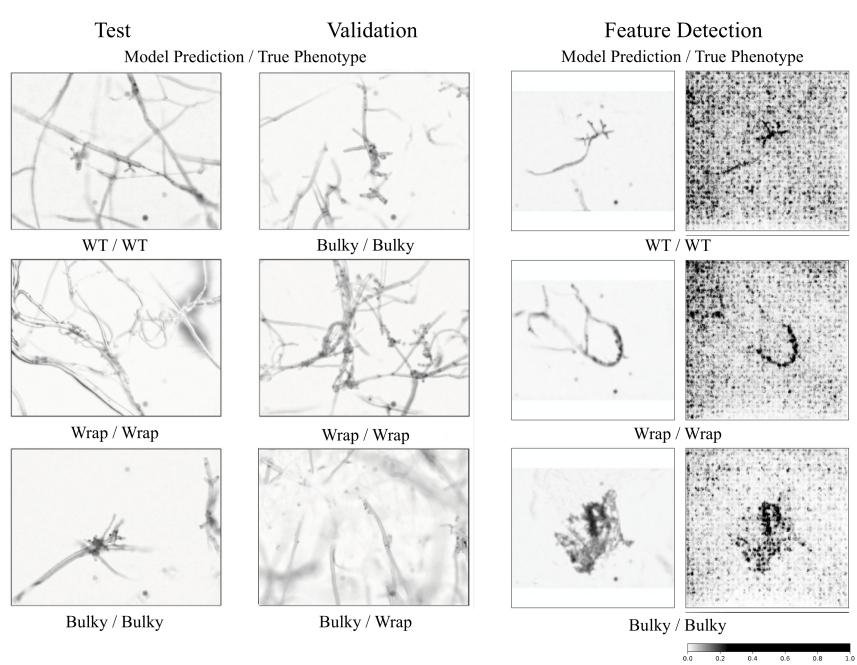

Figure 5. Automatic classification of phenotypes. Model performance on test and validation sets is depicted in the first two columns. The far-left column shows a selected test set, followed by an example validation set in the second column. The third and fourth columns show feature importance evaluation for a few test samples. The third column shows the original image (after padding and resize) with the last column identifying feature importance. A higher value (black) in the last column indicates greater feature importance. All images are labeled with the model predicted phenotype first and manually identified phenotype second. 


\subsection{Crosses Suggest at Least 2-3 Genes Involved in Conidiophore Architectural Phenotypes}

Three crosses were performed between representative strains for each architectural phenotype: FGSC8872 $\times$ FGSC8876 (WT $\times$ Wrap), FGSC8876 $\times$ FGSC2229 (Wrap $\times$ Bulky), and FGSC $8872 \times$ FGSC3943 (WT $\times$ Bulky). A total of 50 progeny were selected from each cross, from which conidiophores were isolated and imaged. For efficiency, these 1932 images were each assigned a phenotype by automatic classification as described above. These phenotype counts were then used to estimate an inheritance model for this discrete complex trait, described below (Table 4).

Table 4. The cell probabilities for the fully epistatic inheritance model with three genes in three crosses. The probability of phenotype $\mathrm{j}$ from cross $\mathrm{i}$ is $\mathrm{K}_{\mathrm{ij}}$.

\begin{tabular}{cccc}
\hline & $\mathbf{A}$ (WT) & B (Wrap) & C (Bulky) \\
\hline $\mathrm{A} \times \mathrm{B}$ & $K_{11}=e^{\lambda} e^{\alpha} e^{-\beta} e^{\alpha \beta}$ & $K_{12}=e^{\lambda} e^{-\alpha} e^{\beta} e^{\alpha \beta}$ & $K_{13}=e^{\lambda} e^{-\alpha} e^{-\beta} e^{-\alpha \beta}$ \\
$\mathrm{B} \times \mathrm{C}$ & $K_{21}=e^{\lambda} e^{-\beta} e^{-\gamma} e^{-\beta \gamma}$ & $K_{22}=e^{\lambda} e^{\beta} e^{-\gamma} e^{\beta \gamma}$ & $K_{23}=e^{\lambda} e^{-\beta} e^{\gamma} e^{\beta \gamma}$ \\
$\mathrm{A} \times \mathrm{C}$ & $K_{31}=e^{\lambda} e^{\alpha} e^{-\gamma} e^{\alpha \gamma}$ & $K_{32}=e^{\lambda} e^{-\alpha} e^{-\gamma} e^{-\alpha \gamma}$ & $K_{33}=e^{\lambda} e^{-\alpha} e^{\gamma} e^{\alpha \gamma}$ \\
\hline
\end{tabular}

The inheritance model is summarized in the table above and motivated by classic models for quantitative traits [26]. In this model, each cross contributes the effect of one dominant allele at each of three loci. For example, in Emericella (Aspergillus) nidulans, at least three genes control conidiophore development [27]. The main effects of the three loci are denoted by $\alpha, \beta$, and $\gamma$ for the A (WT), B (Wrap), and C (Bulky) loci and associated phenotype, respectively. In each cross, there are two dominant alleles (one from each parent) being contributed that epistatically interact to determine the conidiophore architectural phenotype. In principle, these pairwise interactions could result from environmental interactions among progeny in the cross and/or developmental interactions within developing progeny. By isolating progeny in their own agar droplet (see Section 2), the former possibility could be eliminated. The pairwise interactions are denoted by $\alpha \beta, \beta \gamma$, and $\alpha \gamma$. In addition, in this model, there is one grand mean $\lambda$ that is adjusted so that all these probabilities of a progeny phenotype, $\mathrm{K}_{\mathrm{ij}}$, sum to 1 . The data tabled below are the multinomial counts of progeny from each of the three crosses (Table 5):

Table 5. Multinomial counts of progeny phenotypes from each of the three crosses.

\begin{tabular}{cccc}
\hline & A (WT) & B (Wrap) & C (Bulky) \\
\hline $\mathrm{A} \times \mathrm{B}$ & $N_{11}=156$ & $N_{12}=205$ & $N_{13}=253$ \\
\hline $\mathrm{B} \times \mathrm{C}$ & $N_{21}=94$ & $N_{22}=155$ & $N_{23}=275$ \\
\hline $\mathrm{A} \times \mathrm{C}$ & $N_{31}=202$ & $N_{32}=230$ & $N_{33}=198$ \\
\hline
\end{tabular}

A denotes an allele associated with the WT phenotype, B denotes an allele associated with the Wrap phenotype, and $C$ denotes an allele associated with the Bulky phenotype. These counts are stored in a $9 \times 1$ vector $\underline{N}$, the "dependent variable" to be explained by the allelic and epistatic effects. The sum of these nine progeny counts is $N$. Under this model, the log of the expectations of these counts $\mathrm{E}(\mathrm{Y})$ are linear in the parameters of the model, such as the allelic effects; thus, the model is sometimes referred to as a loglinear model [28]. Much as in a probit model, the goal of this discrete trait model is to associate an underlying quantitative trait through the log of the expected counts with the allelic and epistatic effects. Under the simplest hypothesis, all nine of these cell probabilities are equal. Departures from this hypothesis (H0) summarize the variation in the counts as measured by the Pearson $X_{0}^{2}$. Departures from the next simplest hypothesis (H1) summarize the additive variation in the counts as measured by the Pearson $X_{1}^{2}$. Departures from the last, most complicated hypothesis $(\mathrm{H} 2)$ with all three interactions summarizes the additive and epistatic variation by the Pearson $X_{2}^{2}$. The heritability is summarized by the ratio of the additive variation to the total variation: $\mathrm{H} 2=\left(X_{0}^{2}-X_{1}^{2}\right) / X_{0}^{2}$. Many intervening models can be envisioned in a hierarchy of possible inheritance models (Figure 6). 


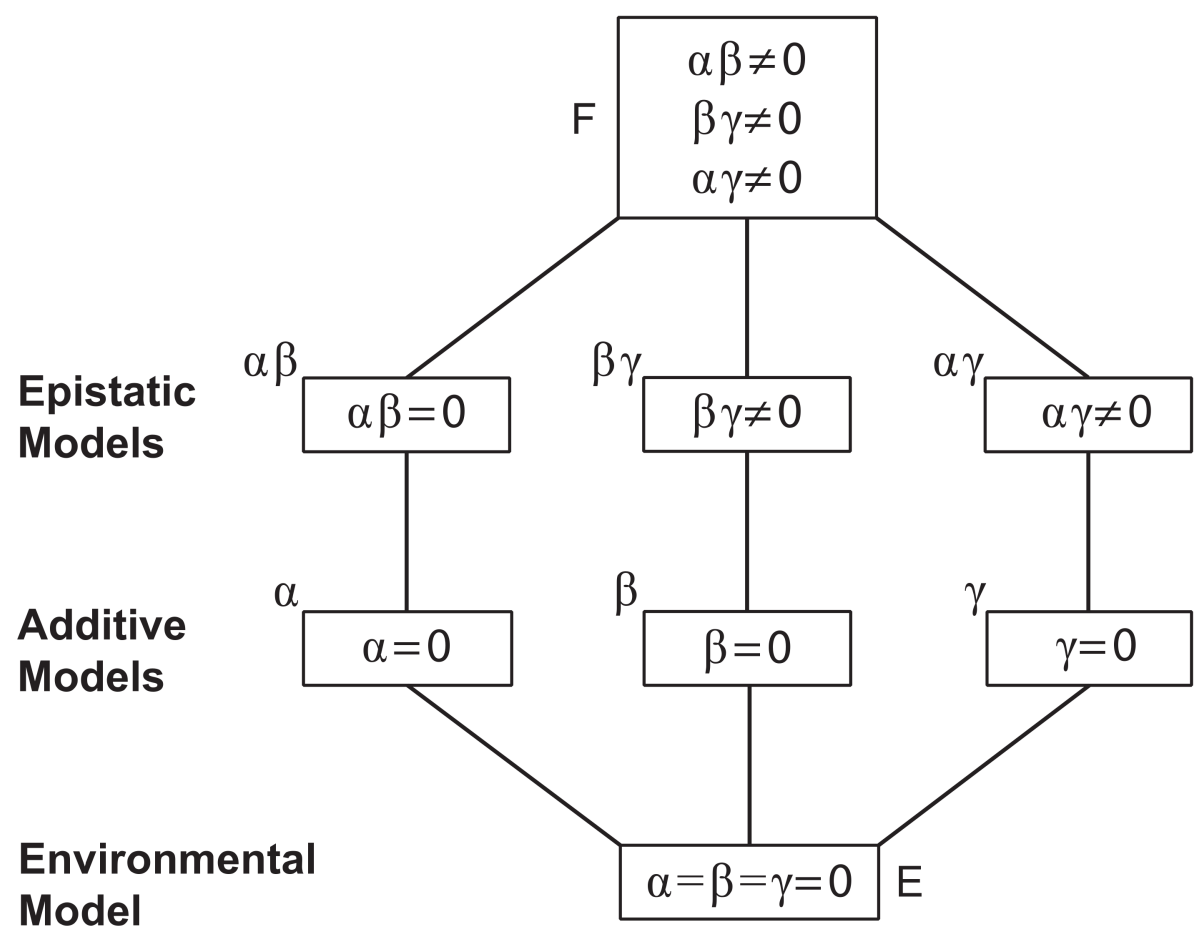

Figure 6. The above hierarchy of models contained particular models that fit the phenotypic counts on three crosses between parents with different conidiophore phenotypes. At the top of the model hierarchy is the full epistatic model (F) with a grand mean, three additive allelic effects, and three epistatic interactions. At the bottom of the hierarchy is an environmental model with no genetic effects on conidiophore phenotype (E). Intervening models in the hierarchy drop one or more parameters describing the additive and epistatic effects of genes $\mathrm{A}, \mathrm{B}$, and $\mathrm{C}$ associated with the conidiophore phenotypes, A, B, and C.

Each model was fitted by the Method of Maximum Likelihood using iteratively reweighted least squares (IRLS) $[29,30]$. Under this approach, the information A about the counts is denoted by A, and the derivatives of the cell probabilities in the model above are stored in the array $\mathrm{X}$ tabled below (Table 6). The matrix NX'AX is the information about the parameters in the model. The score vector $S$ represents the derivatives of the $\log$ of the cell probabilities and can be written as $\mathrm{S}=\mathrm{AY}$, where $\mathrm{A}$ is a $9 \times 9$ diagonal matrix where $\mathrm{A}(\mathrm{i}, \mathrm{i})=1 / \mathrm{K}(\mathrm{i}, \mathrm{i})$.

Table 6. The derivatives of the model cell probabilities are stored in the below $9 \times 7$ derivative matrix $X$. Each row captures the parameters present in an expected cell probability $\mathrm{K}_{\mathrm{ij}}$ of one of the three phenotypes $\mathrm{j}$ in cross $\mathrm{i}$ (Table 4). The $\mathrm{X}$ matrix captures the structure of the model. Dropping/adding columns corresponds to dropping/adding parameters in a model.

\begin{tabular}{cccccccc}
\hline Parameters/K & $\lambda$ & $\alpha$ & $\boldsymbol{\beta}$ & $\boldsymbol{\gamma}$ & $\boldsymbol{\alpha} \boldsymbol{\beta}$ & $\boldsymbol{\beta} \boldsymbol{\gamma}$ & $\boldsymbol{\alpha} \boldsymbol{\gamma}$ \\
\hline$K_{11}$ & 1 & 1 & -1 & 0 & 1 & 0 & 0 \\
$K_{12}$ & 1 & -1 & 1 & 0 & 1 & 0 & 0 \\
$K_{13}$ & 1 & -1 & -1 & 0 & -1 & 0 & 0 \\
$K_{21}$ & 1 & 0 & -1 & -1 & 0 & -1 & 0 \\
$K_{22}$ & 1 & 0 & 1 & -1 & 0 & 1 & 0 \\
$K_{23}$ & 1 & 0 & -1 & 1 & 0 & -1 & 0 \\
$K_{31}$ & 1 & 1 & 0 & -1 & 0 & 0 & 1 \\
$K_{32}$ & 1 & -1 & 0 & -1 & 0 & 0 & -1 \\
$K_{33}$ & 1 & -1 & 0 & 1 & 0 & 0 & 1 \\
\hline
\end{tabular}


The fitting of this model with seven parameters or a simplification of this inheritance model was obtained from solving the below weighted least squares problem where $\mathrm{Y}=\mathrm{S}+\mathrm{NAXN}$ is the provisional quantitative dependent variable, $\mathrm{X}$ is the regression matrix of independent variables, such as allelic effects, $\mathrm{A}$ is the weight matrix, and the parameters are $\beta^{\prime}=(\lambda, \alpha, \beta, \gamma, \alpha \beta, \beta \gamma, \alpha \gamma)$. The parameters are found by solving the following normal equation iteratively:

$$
\mathrm{N} X^{\prime} A X \beta^{*}=\mathrm{X}^{\prime} \mathrm{Y}
$$

The matrix $\mathrm{A}$ and vector $\mathrm{Y}$ are evaluated with the current provisional parameter estimate $\beta$, and then the normal equations are solved for the updated parameter vector $\beta^{*}$. The process is repeated until the relative error is less than $10^{-8}$. The process is initialized by solving the multiple regression problem with $\log \left(\frac{N}{\bar{N}}\right)=X \beta+\epsilon$, where $\epsilon$ is a normally distributed error vector with nine independent components. Goodness of fit was assessed by Pearson $X^{2}$. The only thing to be changed for simplified models is the $X$ matrix by removing the appropriate column(s) to eliminate a parameter or parameters not in a simplified model. The results for fitting the models with IRLS [29] computed with relative error $<10^{-8}$ after nine iterations of IRLS are summarized in the table below.

The full epistatic model with three genes fitted the ratios from three crosses (Table 7). The only epistatic interaction that could possibly be dropped was between the A (WT) and B (Wrap) genes $(\alpha \beta)$. This raised the question of whether or not any one of the additive allelic effects could be dropped altogether. This turned out to be the $\beta$ allelic effect (for Wrap). A model without the B gene for Wrap did fit the progeny counts of the three crosses $\left(X^{2}=9.96, \mathrm{df}=5, p=0.08\right)$. The conclusion was that at least two genes control conidiophore phenotype. An additive model and the model without gene effects was then used to assess the heritability. The heritability of $\mathrm{H}^{2}$ was typical for quantitative traits [26], namely, about 0.23 .

The maximum likelihood estimates of parameters for the inheritance model are tabled (Table 8). The two promising models are fairly consistent across model parameters. One of these models has three loci, and the other has only two loci.

The allelic and epistatic effects are fairly stable between the two models. The C (Bulky) gene appears to have the largest effect both additively and epistatically with both B (Wrap) and A (WT). There also appears to be a strong epistatic interaction between B (Wrap) and C (Bulky) alleles.

Table 7. A nested hierarchy of inheritance models was successfully fitted with at least two genes controlling the conidiophore architectural phenotype to the counts of progeny phenotypes from three crosses (Table 5). Nine iterations were necessary to achieve the desired error tolerance of $10^{-8}$ with iteratively reweighted least square (IRLS). Recommended models are bolded along with their goodness of fit to the counts of phenotypes in crosses. A null hypothesis $(\mathrm{H} 0)$ is tested against an alternative (HA) with the chi-squared test having degrees of freedom (df).

\begin{tabular}{|c|c|c|c|c|c|c|c|}
\hline Model & $X^{2}$ & df & $p$ & $\mathrm{X}^{2}{ }_{\mathrm{HA}}-\mathrm{X}^{2}{ }_{\mathrm{HO}}$ & df & $p$ for HA vs. H0 & Notes \\
\hline Full epistatic & 0.98 & 2 & 0.61 & - & - & - & $\mathrm{H} 0=$ full epistatic \\
\hline$\alpha \beta=0$ & 8.39 & 3 & 0.04 & $8.39-0.98=7.41$ & 1 & 0.004 & $\mathrm{HO}=$ full epistatic \\
\hline$\alpha \beta=\beta=0$ & 9.96 & 5 & 0.08 & $9.96-0.98=8.98$ & 2 & 0.01 & $\mathrm{H} 0=$ full epistatic \\
\hline$\beta \gamma=0$ & 9.32 & 3 & 0.02 & $9.32-0.98=8.34$ & 1 & 0.004 & $\mathrm{H} 0=$ full epistatic \\
\hline$\alpha \gamma=0$ & 84.57 & 3 & $<0.0001$ & $84.57-0.98=83.59$ & 1 & $<0.00001$ & $\mathrm{H} 0=$ full epistatic \\
\hline $\begin{array}{c}\text { environmental } \\
\text { heritability }\end{array}$ & 124.46 & 8 & $<0.0001$ & $124.46-0.98=123.48$ & 7 & $<0.00001$ & $\begin{array}{c}\mathrm{H} 0=\text { full epistatic } \\
\mathrm{H}^{2}=(124.46-95.76) / 124.46=0.23 \\
\mathrm{H} 0=\text { environmental model } \\
\mathrm{H} 1=\text { full additive model }\end{array}$ \\
\hline
\end{tabular}


Table 8. Maximum likelihood estimates of allelic effects and epistatic effects in a two or three locus model of inheritance. The full epistatic model with three genes has three allelic effects and three epistatic interactions. The two-gene model with gene B (Wrap) removed has two allelic effects for A (WT) and C (Bulky) and two epistatic interactions between A (WT) and C (Bulky) and between B (Wrap) and $C$ (Bulky). The standard errors were obtained from the square roots of the diagonal elements of the inverse of the information matrix $N X^{\prime} A X$.

\begin{tabular}{ccc}
\hline Parameters & $\begin{array}{c}\text { Full Epistatic } \\
\text { 3 Genes }\end{array}$ & $\begin{array}{c}\alpha \beta=\beta=0 \\
\text { 2 Genes }\end{array}$ \\
\hline$\lambda$ & $5.32 \pm 0.0040$ & $5.32 \pm 0.0030$ \\
$\alpha$ & $-0.08 \pm 0.0048$ & $0.02 \pm 0.0048$ \\
$\beta$ & $0.05 \pm 0.0061$ & 0 \\
$\gamma$ & $0.53 \pm 0.0047$ & $0.53 \pm 0.0044$ \\
$\alpha \beta$ & $-0.15 \pm 0.0060$ & 0 \\
$\beta \gamma$ & $0.20 \pm 0.0067$ & $0.23 \pm 0.0061$ \\
$\alpha \gamma$ & $-0.60 \pm 0.0064$ & $-0.58 \pm 0.0062$ \\
\hline
\end{tabular}

\subsection{Architectural Phenotype May Impact Colonization Capacity in N. crassa}

Previous work conducted in Aspergillus has shown that conidiophore architecture impacts the colonization capability of the organism [8]. To investigate whether this may also be true in N. crassa, we quantified the average number of conidia produced by each conidiophore phenotype, using a representative strain for each. There was no statistically significant difference in the number of conidia produced by each phenotype when suspended in liquid culture (Figure 7). Additionally, we found no statistically significant difference in average conidium diameter produced by each phenotype (Figure 8).

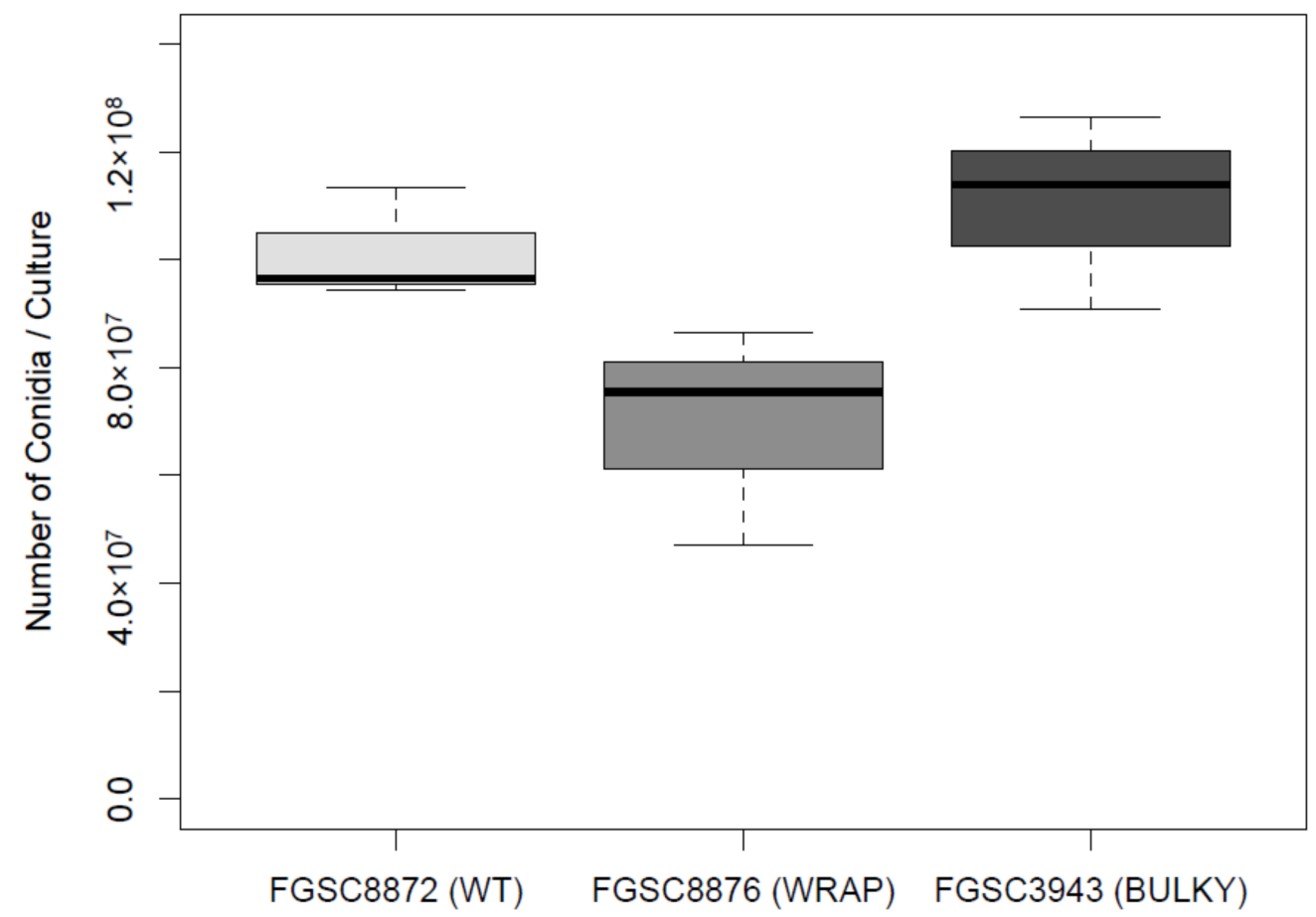

Figure 7. Number of conidia suspended in water from an $82 \mathrm{~mm}$ diameter nitrocellulose membrane by each conidiophore phenotype. The dataset includes three biological replicates. The box shows the 25th and 75th percentiles, with the median denoted as a line inside. The whiskers represent the range of data. The $t$-test results for pairs are as follows: WT-Wrap, $p=0.09541$; Wrap-Bulky, $p=0.06079$; WT-Bulky, $p=0.505$. 


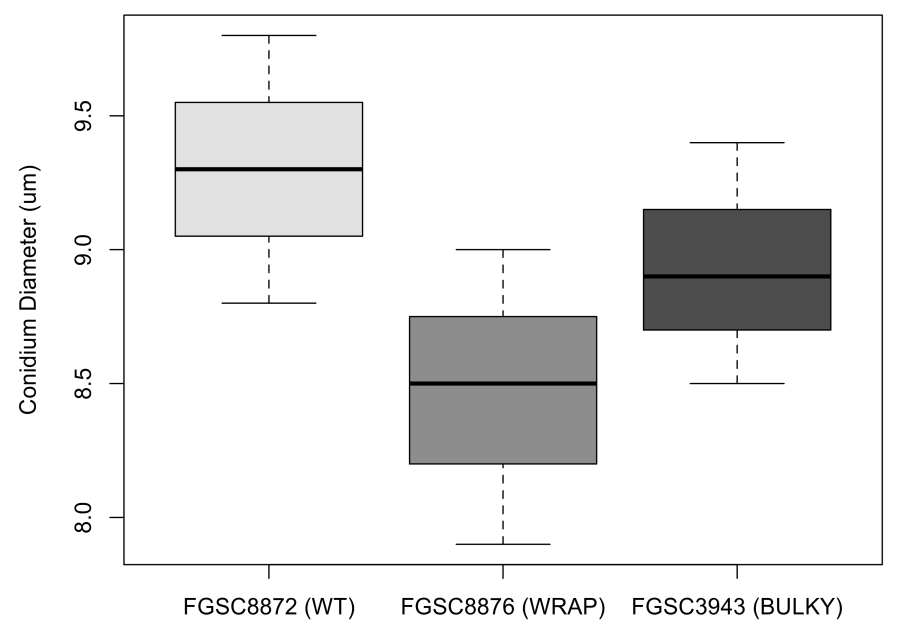

Figure 8. Average conidium diameter in microns when suspended in water for each architectural phenotype. The dataset includes three biological replicates. The box shows the 25th and 75th percentiles, with the median denoted as a line inside. The whiskers represent the range of data. The $t$-test results for pairs are as follows: WT-Wrap, $p=0.125$; Wrap-Bulky, $p=0.3219$; WT-Bulky, $p=0.3995$.

We also explored sporulation behavior on SFG medium, where mature conidiophores were placed at the center of a plate to allow for sporulation and subsequent colonial growth. Interestingly, fewer WT colonies $(n=334)$ developed compared to Wrap and Bulky $(n=1113$ and $n=1252$, respectively), suggesting a difference in sporulation behavior in an aerial versus aqueous environment. We quantified the distance from the center of each nitrocellulose membrane to the center of each colony. The distribution of these distances are referred to as the spore shadow. Two replicate datasets were combined, as there was no significant difference between replicates of a phenotype following a two-sample Kolmogorov-Smirnov test [30]. Sporulation by Wrap conidiophores resulted in colonies at a significantly closer distance when compared to that of Bulky colonies $\left(p=3.101 \times 10^{-5}\right)$ (Figure 9). No significant sporulation distance difference was found for the other pair combinations; however, differences in the distribution of colony distances were found between pairs WT and Wrap $(\mathrm{D}=0.090973$, $p=0.02849)$ and Wrap and Bulky $\left(\mathrm{D}=0.09624, p=3.702 \times 10^{-5}\right)$ by two-sample Kolmogorov-Smirnov tests (Figure 10).

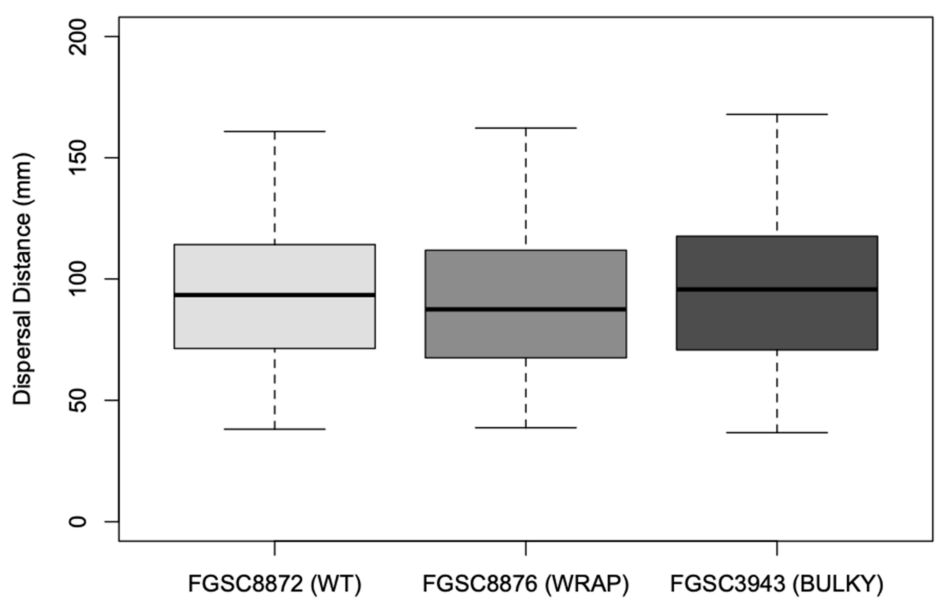

Figure 9. Distance in $\mathrm{mm}$ to the center of each colony on sorbose + fructose + glucose (SFG) medium from the center of each nitrocellulose membrane. The dataset includes two biological replicates. The box shows the 25th and 75th percentiles, with the median denoted as a line inside. The whiskers represent the range of data. The $t$-test results for pairs are as follows: WT-Wrap, $p=0.0587$; Wrap-Bulky, $p=3.101 \times 10^{-5} ;$ WT-Bulky, $p=0.3466$. 


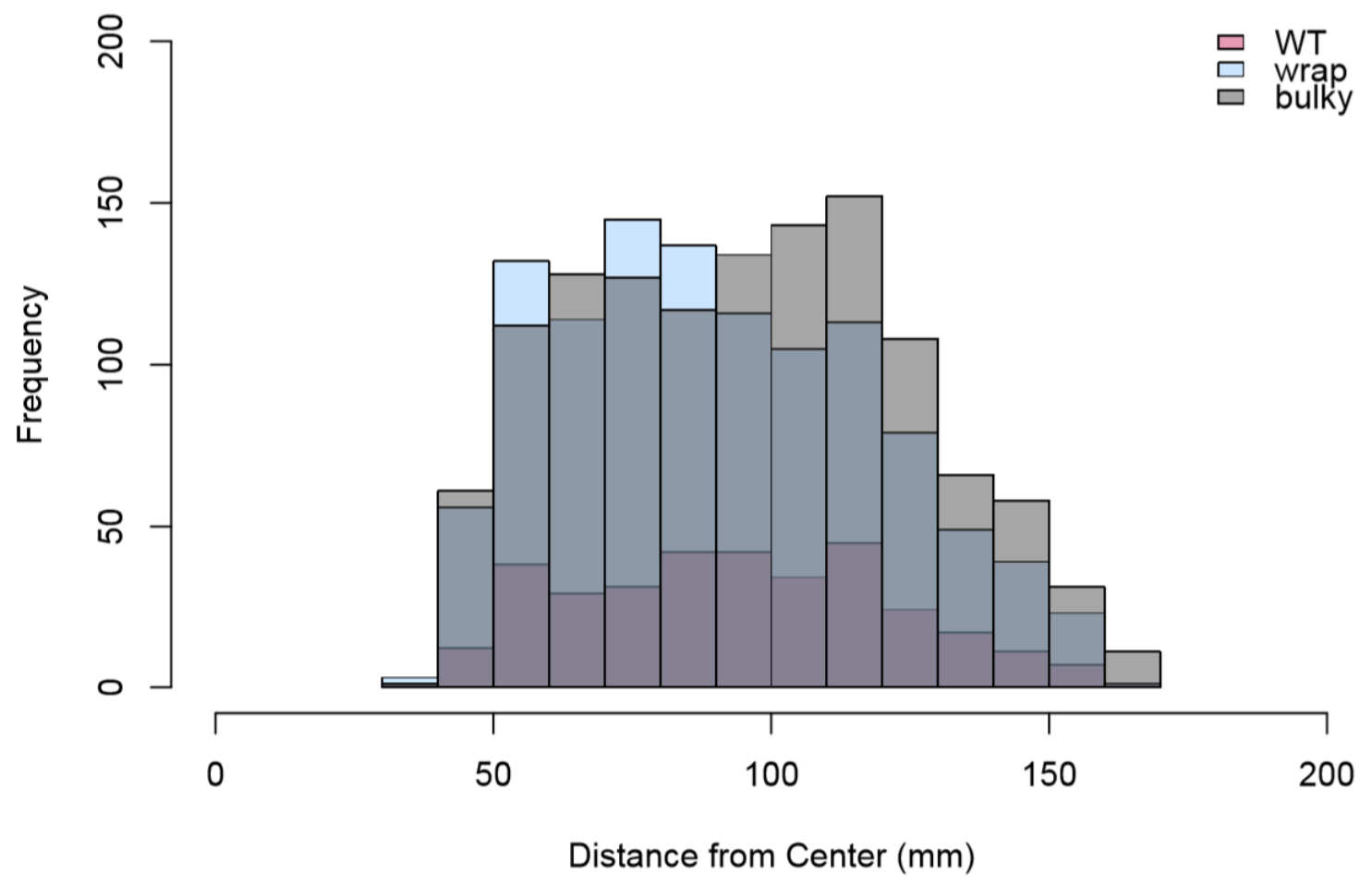

Figure 10. Histogram of distances in $\mathrm{mm}$ to the center of each colony from the center of each nitrocellulose membrane. Total colony counts combining two replicates are as follows: WT $=334$, Wrap $=1113$, Bulky $=1252$. Results of two-sample Kolmogorov-Smirnov tests for each pair are as follows: WT-Wrap D = 0.090973, $p=0.02849$; Wrap-Bulky D = 0.09624, $p=3.702 \times 10^{-5}$; WT-Bulky $\mathrm{D}=0.055465, p=0.3922$.

\section{Discussion}

Conidiophore development in N. crassa has been thoroughly documented over decades of study [31]. Genetic, temporal, and environmental signals guiding this process are well understood [4,31]; however, little is known about conidiophore morphological variation, particularly in natural populations. The N. crassa collection of Louisiana isolates provides a convenient tool for characterizing variation in a natural population and has previously been used to describe local adaptations and reveal novel gene functions [11-13]. Following their collection from nature, these strains underwent conidial plating to remove possible fungal contaminants and were maintained vegetatively at the FGSC [32]. It is entirely likely that these isolates are heterokaryotic, reflective of the genetic variation observed in the wild. By using these populations as is, we aimed to capture the full spectrum of natural variation in conidiophore architecture.

By utilizing this population set of 21 wild strains to explore morphological variation in the conidiophore, we identified three novel architectural phenotypes: Wild Type, Bulky, and Wrap. These three phenotypes are consistently displayed throughout conidiophore development and are likely not the result of temporal disparity along the same growth trajectory. Interestingly, no clear dependence of phenotype on collection location or substrate was observed, suggesting a genetic component to this variation. To further explore this, we conducted crosses between representative strains for each phenotype. By quantifying the subsequent progeny conidiophores of these crosses, we were able to fit a model for inheritance. Our resulting model suggests that at least two genes control conidiophore architectural phenotype with an estimated heritability of 0.23 .

Future work should seek to identify these genetic variants and characterize transcriptional profiles for conidiophores of different architectural phenotypes. Many key genetic players of conidiophore development have already been identified and thoroughly characterized, most notably the con genes [33], 
and transcriptomic data have elucidated gene expression profiles throughout wild-type conidiophore development [7]. Interestingly, the Bulky conidiophore phenotype resembles that of the attenuated (at) knockout mutant, where conidia form in dense aggregates [34]. However, we do not observe slowed growth and pigmentation in the Bulky wild isolates as is seen in at mutants. Identifying and characterizing additional genes possibly underlying WT, Bulky, and Wrap phenotypes would provide greater understanding of conidiophore development through a morphological lens and perhaps lend insight into the natural population structure of these wild isolates.

To investigate a potential environmental impact of conidiophore architecture, we evaluated the effect of conidiophore phenotype on sporulation in both an aqueous suspension and aerial environment. No significant effect was detected on the number of conidia or average conidium diameter when suspended in water, nor was there any difference in wettability of conidia while washing off a plate. This indicates that the conidiophore architectural phenotype is not due to difference in hydrophobicity of the conidia. In an aerial environment, fewer conidia from Wrap conidiophores germinated compared to Bulky. Additionally, spore shadows by conidia from Wrap conidiophores fit a different distribution compared to that of both WT and Bulky groups. This suggests that conidiophore architectural phenotype may impact colonization capacity of the organism. Sporulation experiments should be conducted at a larger scale to more effectively determine the maximum dispersal distance of each conidiophore phenotype and, hence, effective population size and neighborhood size $[35,36]$. Furthermore, it is possible that conidiophore phenotype may contribute to microspatial variation through altering effective population size and neighborhood size, as some strains exhibiting different architectural phenotypes were collected from the same substrate in the same location [37].

It remains a challenge to relate complex traits describing growth and form to their underlying genes [1]. Doing so requires genomics [13,38], transcriptomics [12], and/or metabolomics [39], as well as high-throughput approaches to phenotyping. Morphological diversity presents an attractive opportunity for image analyses and phenotyping at a large scale, as shown in other model fungal systems such as yeast [40,41]. Phenomics tools like Digital Imaging of Root Traits (DIRT) have been developed specifically to quantify architectural features in plant root systems [42]. Although filamentous fungi bear some similarities to roots, the unique morphology of conidiophores is best characterized with a tool specifically designed for their structure. We presented a novel method to classify brightfield images of conidiophores into three architectural phenotypes and extract important features for their classification. This same approach can be applied to characterize conidiophores of other filamentous fungi and may even be used in conjunction with DIRT to characterize morphology in the symbiotic relationship between roots and arbuscular mycorrhiza [43,44]. To further improve our method, automation in sample collections and blurred region removal could be implemented. Performance in model training was also limited by the sample size in our study and domain-specific features.

Author Contributions: Conceptualization, E.K.K., M.S., and J.A.; data curation, E.K.K.; formal analysis, E.K.K., Y.W., M.S., and J.A.; funding acquisition, E.K.K., L.M., and J.A.; investigation, E.K.K. and Y.W.; methodology, E.K.K., Y.W., M.S., L.M., and J.A.; project administration, L.M. and J.A.; resources, L.M. and J.A.; software, Y.W.; supervision, L.M. and J.A.; validation, L.M. and J.A.; visualization, E.K.K. and Y.W.; writing-original draft, E.K.K.; writing-review and editing, Y.W., M.S., L.M., and J.A. All authors have read and agreed to the published version of the manuscript.

Funding: This research was funded by the National Science Foundation (MCB-1713746), the US Department of Energy (DE-FOA-0002214), and the Achievement Rewards for College Scientists Foundation (2018-2019-ATL).

Acknowledgments: We acknowledge Mary E. Case for suggesting the two-gene hypothesis in the heritability study. We also thank James Griffith and the UGA GACRC.

Conflicts of Interest: The authors declare no conflict of interest. The funders had no role in the design of the study; in the collection, analyses, or interpretation of data; in the writing of the manuscript, or in the decision to publish the results. 


\section{Appendix A}

Table A1. Population of wild Louisiana isolates of N. crassa. Strains used in this study were obtained from the FGSC. Mating type (Mat) of each strain is denoted by A or a.

\begin{tabular}{ccccccc}
\hline Strain Number & FGSC & Perkins Mat & Strain Provenance & Collection Site & Substrate/Annotation \\
\hline Wild Strains & & & & & & \\
D110 & 8870 & 4448 & A & Dettman, J. & Franklin, LA & sugarcane \\
D111 & 8871 & 4449 & a & Dettman, J. & Franklin, LA & sugarcane \\
D112 & 8872 & 4453 & A & Dettman, J. & Franklin, LA & sugarcane \\
D114 & 8874 & 4464 & A & Dettman, J. & Franklin, LA & sugarcane \\
D116 & 8876 & 4481 & a & Dettman, J. & Franklin, LA & sugarcane \\
D118 & 8878 & 4491 & a & Dettman, J. & Franklin, LA & sugarcane \\
JW09 & 2229 & & A & Welch, J. & Welsh, LA & burned grass \\
JW10 & 2229 & & A & Welch, J. & Welsh, LA & burned grass \\
JW59 & 3200 & & a & Welch, J. & Coon, LA & burned stumps \\
JW66 & 3211 & & a & Welch, J. & Sugartown, LA & Pine burn \\
JW70 & 3199 & & A & Welch, J. & Coon, LA & burned stumps \\
JW75 & 3943 & & a & Welch, J. & Houma, LA & sugarcane burn \\
& 847 & & A & Lein & Louisiana & sugarcane burn \\
D113 & 8873 & 4454 & a & Dettman, J. & Franklin, LA & sugarcane \\
D119 & 8879 & 4500 & a & Dettman, J. & Franklin, LA & sugarcane \\
JW20 & 3212 & & A & Welch, J. & Ravenswood, LA & bonfire \\
JW76 & 3943 & & a & Welch, J. & Houma, LA & sugarcane burn \\
JW159 & 2221 & & a & Welch, J. & Houma, LA & sugarcane burn \\
JW160 & 2222 & & A & Welch, J. & Iowa, LA & grass burn \\
JW162 & 2223 & 2224 & a & Welch, J. & Iowa, LA & grass burn \\
JW164 & 2228 & a & Welch, J. & Marrero, LA & wood burn \\
JW167 & 2489 & A & Welch, J. & Roanoke, LA & grass burn \\
OR74A & & & FGSC & Marrero, LA & unknown \\
\hline
\end{tabular}

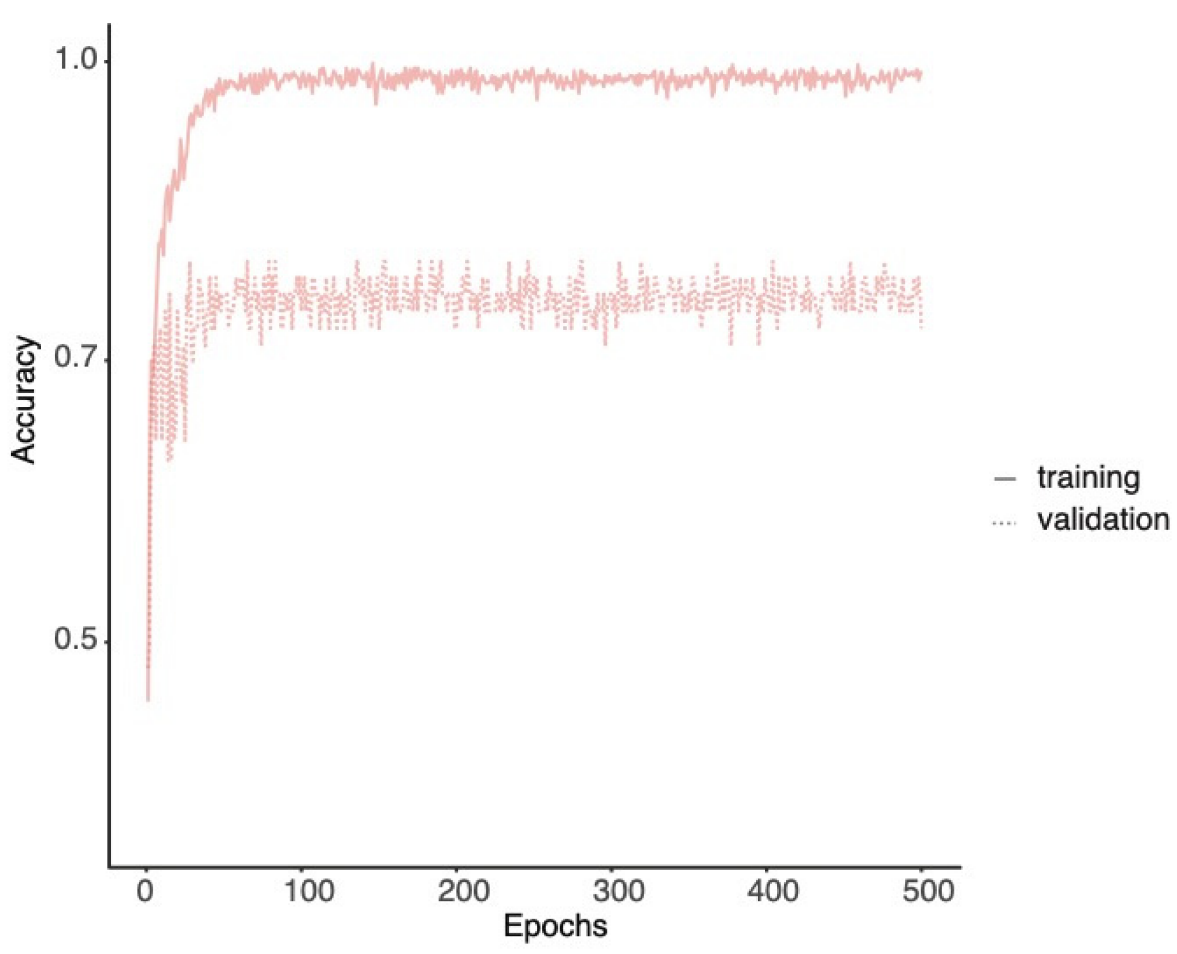

Figure A1. Model accuracies through training. Accuracies of the training (solid line) and validation (dotted line) sets is plotted through epochs. The final model was chosen on the basis of the accuracy of the validation set. 


\section{References}

1. Davis, R.H. Contributions of a Model Organism; Oxford University Press: New York, NY, USA, 2000.

2. Maheshwari, R. Microconidia of Neurospora crassa. Fungal Genet. Biol. 1999, 26, 1-18. [CrossRef]

3. Sargent, M.L.; Kaltenborn, S.H. Effects of Medium Composition and Carbon Dioxide on Circadian Conidiation in Neurospora. Plant Physiol. 1972, 50, 171-175. [CrossRef]

4. Springer, M.L.; Yanofsky, C. A morphological and genetic analysis of conidiophore development in Neurospora crassa. Genes Dev. 1989, 3, 559-571. [CrossRef]

5. Olmedo, M.; Ruger-Herreros, C.; Corrochano, L.M. Regulation by Blue Light of the fluffy Gene Encoding a Major Regulator of Conidiation in Neurospora crassa. Genetics 2009, 184, 651-658. [CrossRef]

6. Bailey-Shrode, L.; Ebbole, D.J. The fluffy Gene of Neurospora crassa is Necessary and Sufficient to Induce Conidiophore Development. Genetics 2004, 166, 1741-1749. [CrossRef]

7. Greenwald, C.J.; Kasuga, T.; Glass, N.L.; Shaw, B.D.; Ebbole, D.J.; Wilkinson, H.H. Temporal and Spatial Regulation of Gene Expression During Asexual Development of Neurospora crassa. Genetics 2010, 186, 1217-1230. [CrossRef] [PubMed]

8. Wang, F.; Dijksterhuis, J.; Wyatt, T.; Wösten, H.A.B.; Bleichrodt, R.-J. VeA of Aspergillus niger increases spore dispersing capacity by impacting conidiophore architecture. Antonie van Leeuwenhoek 2015, 107, 187-199. [CrossRef]

9. Lau, G.W.; Hamer, J.E. Acropetal: A Genetic Locus Required for Conidiophore Architecture and Pathogenicity in the Rice Blast Fungus. Fungal Genet. Biol. 1998, 24, 228-239. [CrossRef]

10. Mir-Rashed, N.; Jacobson, D.; Dehghany, M.; Micali, O.; Smith, M. Molecular and Functional Analyses of Incompatibility Genes at het-6 in a Population of Neurospora crassa. Fungal Genet. Biol. 2000, 30, 197-205. [CrossRef]

11. Ellison, C.E.; Hall, C.; Kowbel, D.; Welch, J.; Brem, R.B.; Glass, N.L.; Taylor, J.W. Population genomics and local adaptation in wild isolates of a model microbial eukaryote. Proc. Natl. Acad. Sci. USA 2011, 108, 2831-2836. [CrossRef]

12. Ellison, C.E.; Kowbel, D.; Glass, N.L.; Taylor, J.W.; Brem, R.B. Discovering Functions of Unannotated Genes from a Transcriptome Survey of Wild Fungal Isolates. mBio 2014, 5, e01046-13. [CrossRef]

13. Palma-Guerrero, J.; Hall, C.R.; Kowbel, D.; Welch, J.; Taylor, J.W.; Brem, R.B.; Glass, N.L. Genome Wide Association Identifies Novel Loci Involved in Fungal Communication. PLoS Genet. 2013, 9, e1003669. [CrossRef]

14. He, K.; Zhang, X.; Ren, S.; Sun, J. Deep Residual Learning for Image Recognition. In Proceedings of the 2016 IEEE Conference on Computer Vision and Pattern Recognition, Las Vegas, NV, USA, 27-30 June 2016; pp. 770-778.

15. McCluskey, K. The Fungal Genetics Stock Center: From Molds to Molecules. Adv. Appl. Microbiol. 2003, 52, $245-262$. [CrossRef] [PubMed]

16. Schneider, C.A.; Rasband, W.S.; Eliceiri, K.W. NIH Image to ImageJ: 25 years of image analysis. Nat. Methods 2012, 9, 671-675. [CrossRef]

17. Deng, Z.; Arsenault, S.; Caranica, C.; Griffith, J.; Zhu, T.; Al-Omari, A.; Schüttler, H.-B.; Arnold, J.; Mao, L. Synchronizing stochastic circadian oscillators in single cells of Neurospora crassa. Sci. Rep. 2016, 6, 35828. [CrossRef]

18. Russakovsky, O.; Deng, J.; Su, H.; Krause, J.; Satheesh, S.; Ma, S.; Huang, Z.; Karpathy, A.; Khosla, A.; Bernstein, M.; et al. ImageNet Large Scale Visual Recognition Challenge. Int. J. Comput. Vis. 2015, 115, 211-252. [CrossRef]

19. Sokolova, M.; Lapalme, G. A systematic analysis of performance measures for classification tasks. Inf. Process. Manag. 2009, 45, 427-437. [CrossRef]

20. Paszke, A.; Gross, S.; Massa, F.; Lerer, A.; Bradbury, J.; Chanan, G.; Killeen, T.; Lin, Z.; Gimelshein, N.; Antiga, L.; et al. PyTorch: An Imperative Style, High-Performance Deep Learning Library. arXiv 2019, arXiv:1912.01703. 
21. Sundararajan, M.; Taly, A.; Yan, Q. Axiomatic Attribution for Deep Networks. arXiv 2017, arXiv:1703.01365.

22. Adebayo, J.; Gilmer, J.; Muelly, M.; Goodfellow, I.; Hardt, M.; Kim, B. Sanity Checks for Saliency Maps. In Proceedings of the 32nd Conference on Neural Information Processing Systems (NeurIPS 2018), Montréal, QC, Canada, 3-8 December 2018.

23. Sturmfels, P.; Lundberg, S.; Lee, S.-I. Visualizing the Impact of Feature Attribution Baselines. Distill 2020, 5, e22. [CrossRef]

24. Case, M.E.; Griffith, J.; Dong, W.; Tigner, I.L.; Gaines, K.; Jiang, J.C.; Jazwinski, S.M.; Arnold, J. The aging biological clock in Neurospora crassa. Ecol. Evol. 2014, 4, 3494-3507. [CrossRef]

25. Brunson, J.K.; Griffith, J.; Bowles, D.; Case, M.E.; Arnold, J. lac-1 and lag-1 with ras-1 affect aging and the biological clock in Neurospora crassa. Ecol. Evol. 2016, 6, 8341-8351. [CrossRef]

26. Falconer, D.S. Introduction to Quantitative Genetics; Longman: London, UK, 1981.

27. Timberlake, W.E. Molecular Genetics of Aspergillus Development. Annu. Rev. Genet. 1990, $24,5-36$. [CrossRef]

28. Williams, C.J.E.A. An Analysis of Density-Dependent Viability Selection. J. Am. Stat. Assoc. 1989, 84, 662-668. [CrossRef]

29. Asmussen, M.A.; Arnold, J.; Avise, J.C. Definition and Properties of Disequilibrium Statistics for Associations between Nuclear and Cytoplasmic Genotypes. Genetics 1987, 115, 755-768.

30. Kendall, M.; Stuart, A. The Advanced Theory of Statistics. Vol.2: Inference and Relationship; Macmillan: New York, NY, USA, 1979.

31. Park, H.-S.; Yu, J.-H. Genetic control of asexual sporulation in filamentous fungi. Curr. Opin. Microbiol. 2012, 15, 669-677. [CrossRef]

32. Turner, B.C.; Perkins, D.D.; Fairfield, A. Neurospora from Natural Populations: A Global Study. Fungal Genet. Biol. 2001, 32, 67-92. [CrossRef]

33. Berlin, V.; Yanofsky, C. Protein changes during the asexual cycle of Neurospora crassa. Mol. Cell. Biol. 1985, 5, 839-848. [CrossRef]

34. Perkins, D.D.; Radford, A.; Sachs, M.S. The Neurospora Compendium: Chromosomal Loci; Academic Press: San Diego, CA, USA, 2000.

35. Powell, J.R.; Dobzhansky, T. How Far Do Flies Fly? The effects of migration in the evolutionary process are approached through a series of experiments on dispersal and gene diffusion in Drosophila. Am. Sci. 1976, 64, 179-185.

36. Lemke, K. Dispersal Models for Drosophila. Master's Thesis, Statistics Department, University of Georgia, Athens, GA, USA, 1985.

37. Powell, A.J.; Jacobson, D.J.; Salter, L.; Natvig, D.O. Variation among natural isolates of Neurospora on small spatial scales. Mycologia 2003, 95, 809-819. [CrossRef]

38. Galagan, J.E.; Calvo, S.E.; Borkovich, K.A.; Selker, E.U.; Read, N.D.; Jaffe, D.; Fitzhugh, W.; Ma, L.-J.; Smirnov, S.; Purcell, S.; et al. The genome sequence of the filamentous fungus Neurospora crassa. Nat. Cell Biol. 2003, 422, 859-868. [CrossRef] [PubMed]

39. Judge, M.T.; Wu, Y.; Tayyari, F.; Hattori, A.; Glushka, J.; Ito, T.; Arnold, J.; Edison, A.S. Continuous in vivo Metabolism by NMR. Front. Mol. Biosci. 2019, 6, 26. [CrossRef]

40. Ohya, Y.; Sese, J.; Yukawa, M.; Sano, F.; Nakatani, Y.; Saito, T.L.; Saka, A.; Fukuda, T.; Ishihara, S.; Oka, S.; et al. High-dimensional and large-scale phenotyping of yeast mutants. Proc. Natl. Acad. Sci. USA 2005, 102, 19015-19020. [CrossRef]

41. Levy, S.F.; Siegal, M.L. Network Hubs Buffer Environmental Variation in Saccharomyces cerevisiae. PLoS Biol. 2008, 6, e264. [CrossRef]

42. Das, A.; Schneider, H.; Burridge, J.; Ascanio, A.K.M.; Wojciechowski, T.; Topp, C.N.; Lynch, J.P.; Weitz, J.S.; Bucksch, A. Digital imaging of root traits (DIRT): A high-throughput computing and collaboration platform for field-based root phenomics. Plant Methods 2015, 11, 1-12. [CrossRef] [PubMed] 
43. Parniske, M. Arbuscular mycorrhiza: The mother of plant root endosymbioses. Nat. Rev. Genet. 2008, 6, 763-775. [CrossRef]

44. Johnson, N.C.; Hoeksema, J.D.; Chaudhary, V.B.; Gehring, C.; Klironomos, J.; Moutoglis, P.; Simard, S.; Swenson, W.; Umbanhowar, J.; Zabinski, C.; et al. From Lilliput to Brobdingnag: Extending Models of Mycorrhizal Function across Scales. Bioscience 2006, 56, 889-900. [CrossRef]

Publisher's Note: MDPI stays neutral with regard to jurisdictional claims in published maps and institutional affiliations.

(C) 2020 by the authors. Licensee MDPI, Basel, Switzerland. This article is an open access article distributed under the terms and conditions of the Creative Commons Attribution (CC BY) license (http://creativecommons.org/licenses/by/4.0/). 